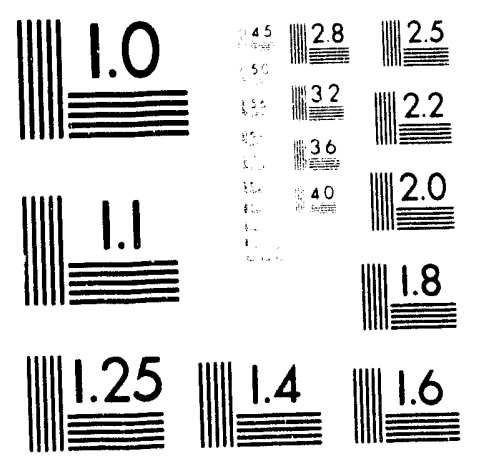



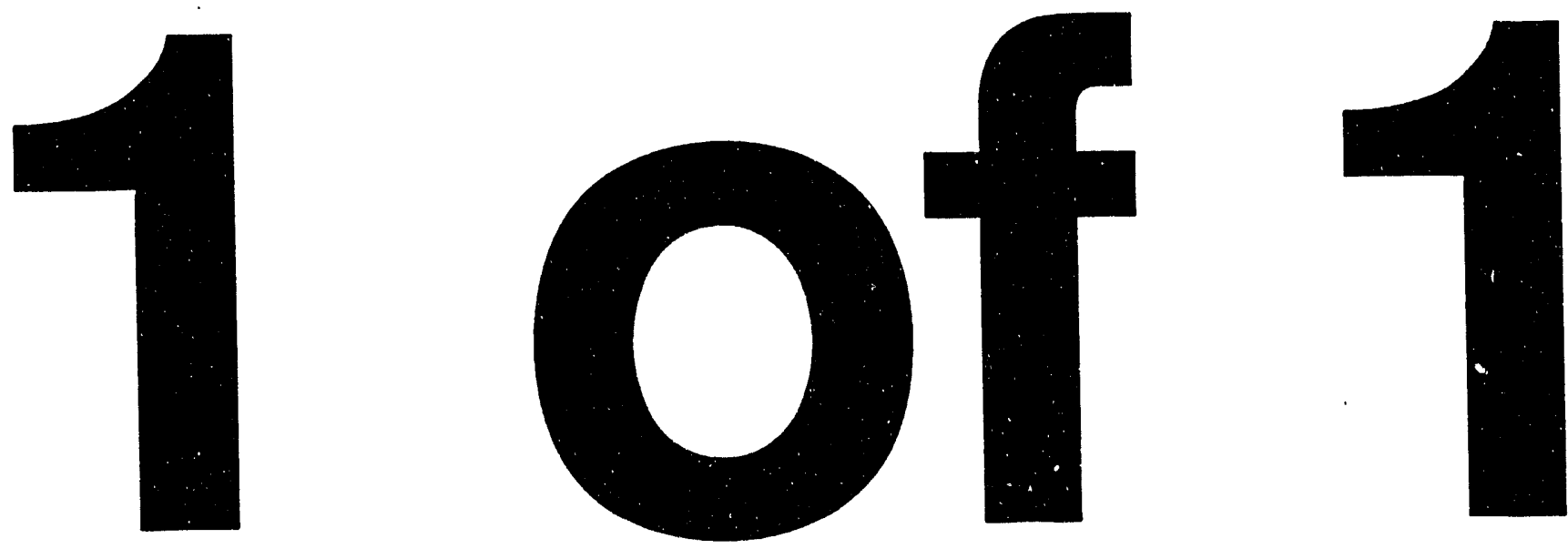


\title{
Analysis of Mechanical Fabrication Experience with CEBAF's Production SRF Cavities*
}

\author{
J. Mammosser, P. Kneisel and J. F. Benesch \\ Continuous Electron Beam Accelerator Facility \\ 12000 Jefferson Avenue, Newport News, Virginia 23606 USA
}

\begin{abstract}
CEBAF has received a total of 360 five-cell niobium cavities, the largest group of industrially fabricated auperconducting cavities $s 0$ far. An extensive data base exists on the fabrication, surface treatment, assembly and cavity performance parameters. Analysis of the mechanical features of the cavities includes the following: the spread in fabrication tolerances of the cells derived from field profiles of the "as fabricated" cavities and the "as fabricated" external $Q$-values of the fundamental power coupler compared to dimensional deviations. A comparison is made of the pressure sensitivity of cavities made of materials from different manufactureas between 760 torr $(4.2 \mathrm{~K})$ and 23 torr $(2 \mathrm{~K})$.
\end{abstract}

\section{INTRODUCTION}

The Continuous Electron Beam Accelerator Facility presently under construction in Newport News, VA is scheduled to produce a bw emittance $200 \mu \mathrm{A}$ eloctron beam of $4 \mathrm{GeV}$ for experimental studies in nuclear physics by mid1994. This beam is accelerated by a string of 338, 5-cell, $1500 \mathrm{MHz}$ niobium cavities arranged in two anti-parallel linear accelerators and operating above design gradients of 5 $\mathrm{MV} / \mathrm{m}$ and $Q_{0}$ values of $2.4 \times 10^{9}$ at $2 \mathrm{~K}$. This system will represent the largest assembly of supenconducting accelerator cavities in the US, and a large data base already exists on the performance of these cavities. Industry has manufactured 360 cavities in accordance with CEBAFs specifications [1] and the superconducting properties of these cavities exceed the design values of field and $Q_{0}$ value by a factor of about 2 to 3 [2]. Most of these cavities have been inspected and evaluated on the basis of compliance with mechanical specifications; about $60 \%$ have been tested, and data are available on frequency variations during chemical processing and pressure sensitivity between 760 torr and 23 torr. In the following eections the current experiences with the above-mentioned feanures of the cavities are discussed.

\section{MECHANICAL FABRICATION}

\section{A. Caviry Tolerances}

The CEBAF/Comell 5-cell cavity has been specified in CEBAFs "Statement of Work" based on earlier experiences gained at Cornell University [3]. Besides very detailed prescriptions for material inspections, material handling and chemical treatment and requirements for electron beam welding, this document also specifies the frequency of the "as-

\footnotetext{
"This work was mpponed by DOE Contraa DE-ACOS-84ER40150.
}

manufactured" cavity as $f=(1494.7 \pm 2) \mathrm{MHz}$, which in turn determines the wlerance on the overall length to $\pm 6.5 \mathrm{~mm}$. Since the cavity cells are deep drawn from $3.2 \mathrm{~mm}$ thick niobium sheet, these tolerances represent a certain challenge to the industrial partner because of spring back, nonuniformity of the starting material and possible variations in weld shrinkage during the electron beam welding of the cavity subsystems. The manufacturer was asked to provide frequency and field profile measurements before and after final tuning and machining of the cavities. From these data the frequency errors of each individual cell of each cavity can be oblained by making use of a lumped circuit analysis of coupled resonators ("tuning program") [4].

This work supported by the U.S. Deparment of Energy under

Figure 1 shows the frequency spread of the "asmanufactured" cavities. The cells were manufactured so that the mean frequency is approximately $1495.4 \mathrm{MHz}$, which is greater than the desired frequency. The cavities were tuned by the manufacturer, all in the same direction. Typical stored energy distribution of as-manufactured cavities is shown by frequency errors in Table 1 . In most of the cases the stored energy was concentrated in the center cells which allowed the manufacturer to restrict tuning to these cells to obtain equal stored energy in all cells $( \pm 2.5 \%)$.

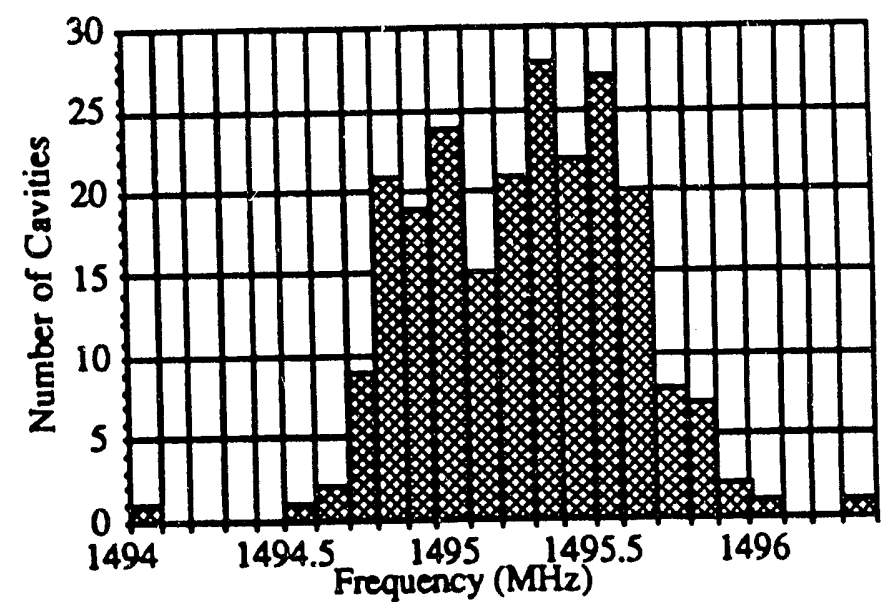

Figure 1. As-received cavity frequencies.

Table 1

\begin{tabular}{|c|c|c|c|c|c|}
\hline \multicolumn{6}{|c|}{ Frequency Errors in $\mathrm{kHz}$ of Each Cell as Manufactured } \\
\hline & Cell\#1 & Cell\#2 & Cell\#3 & Cell \#4 & Cell\#5 \\
\hline an & -610 & 1101 & 1464 & 1100 & -331 \\
\hline tod dev & 455 & 628 & 577 & 637 & 486 \\
\hline & -1760 & .1440 & -726 & -1318 & .1375 \\
\hline & 1906 & 4439 & 4279 & 4439 & 1227 \\
\hline
\end{tabular}


The spread of the cell-to-cell coupling coefficiem $k$ as derived from the frequencies of the fundamental passband modes according to

$$
k=\frac{f(x)^{2}-f(x / 5)^{2}}{\int(x)^{2}+\left(\int(x / S)^{2} \cos (x / s)\right)}
$$

is shown in Figure 2. It can be seen that the cell-to-cell coupling varies, depending on the amount of tuning which had to be done in the $\pi$-mode for each individual cell. This variation in $k$ causes a desirable spread in the frequencies of the higher-order modes, increasing the instability threshold of the socelerator [5].

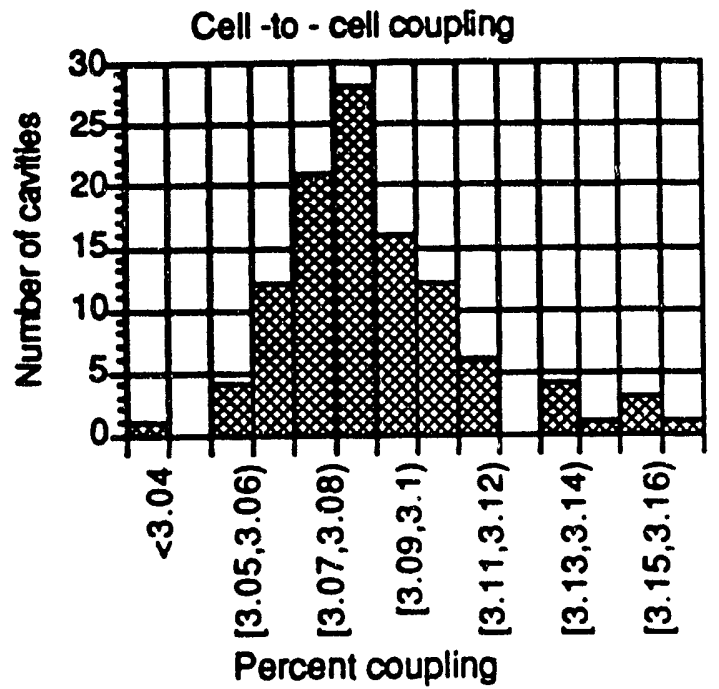

Figure 2. Variations in cell-to-cell coupling.

\section{B. Fundamental Power Coupler (FPC)}

The coupling of the of power into the CEBAF/Cornell cavity is provided by a waveguide systern shorted at one end. The coupling strength has been selected at $Q_{\text {ext }}=6.6 \times 10^{6}$. $\pm 20 \%$. This strength of the coupling is 0.8 times the value required for critical coupling at full beam. This value of coupling permits adequate control bandwidth, requires somewhat less klystron power at less than full current than would critical coupling at full beam, and permits operation at higher gradients with the available if power than would critical coupling at full beam. The coupling of the if to the cavity fields is established by the linkage of a standing wave in the input coupler waveguide and the cavity fields. Figure 3 shows the standing wave pattern as measured by a perturbation measurement for two different cases of $Q$ ext: a less strong coupling is achieved by shifting the standing wave in the region of the iris opening of the end cell, which can be acomplished by varying the distance of the waveguide short to beam axis.

Similarly, the coupling strength can be decreased by equeczing the input waveguide or increased by opening up the waveguide at the point of the highest stored energy [6]. This method is used for final adjustment of the $Q$ ext value. As a practical matter the value of $Q_{\text {ext }}$ is very sensitive to mechanical dimensions of the waveguide arrangement, as is
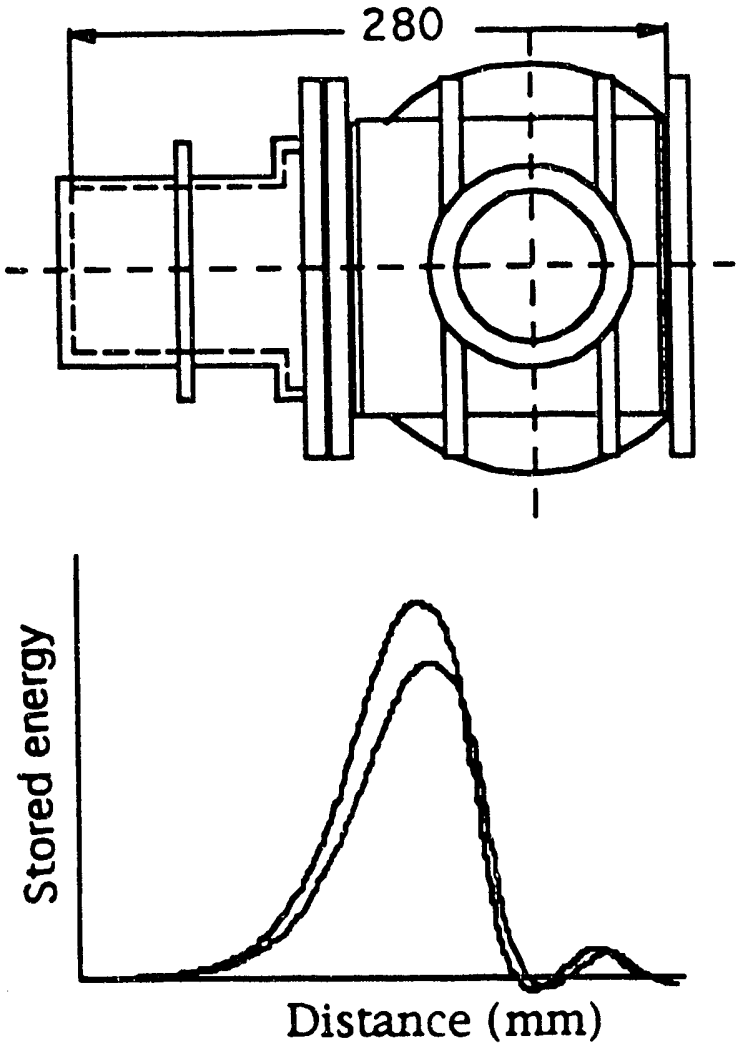

Figure 3. Standing wave measured in FPC with bead pull on FPC axis for two different $Q$ ext values (upper trace $Q_{\mathrm{ext}}=8.6 \mathrm{E} 6$, lower trace $Q_{\mathrm{ext}}=6 \mathrm{E} 6$ ).

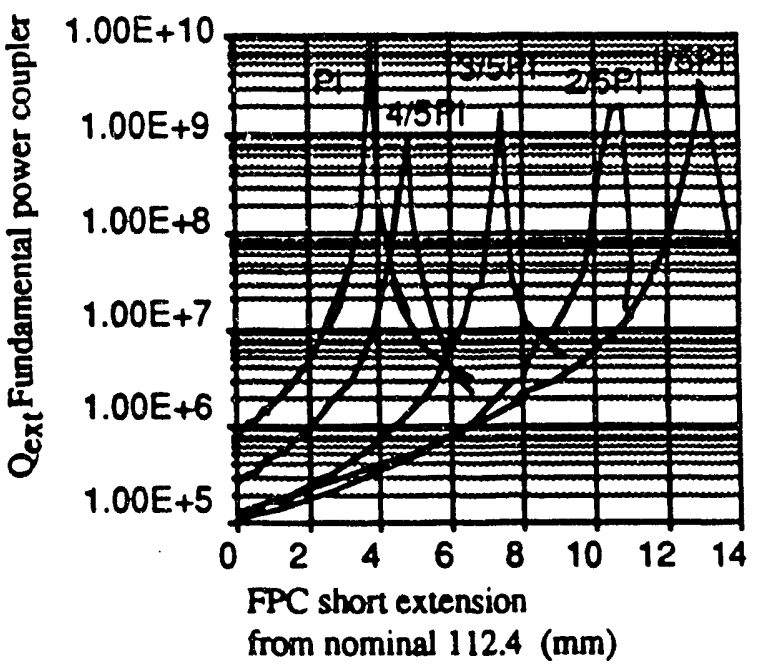

Figure 4. Qext as a function of shor distance to beam axis.

shown in Figure 4. Here the Qext values for all 5 passband modes are plotted as a function of the short distance to the beam axis.

According to these measurements, a fixed dimension of $114.6 \mathrm{~mm}$ will provide the desired design value for $Q_{\text {exl. }}$. Any 
deviation from this number is caused by fabrication tolerances such as weld shrinkage, warpage, deflections of the waveguide or machining tolerances. The $Q_{\text {ext }}$ values of 329 "asfabricated" cavities have an average of 7.55E6. Fortunately, in most cases the desired value can be achieved easily by slight mechanical deformations of the waveguide as mentioned above.

\section{FREQUENCY SHIFTS}

\section{A. Chemical Treatment of the Cavities}

When delivered by the manufacturer the cavities have received only a very slight material removal for cleaning purposes after the firal welding and machining steps. The major chemical treatments are done at CEBAF. It has been extablished [3] that at least a layer of 50 um ("surface damage hyer") has to be removed from the surface to achieve or exceed CEBAF's design values for $Q 0$ value and accelerating gradient Assuming uniform material removal from the surface by immersing the whole cavity into an acid bath of equal parts of hydrofluoric (48\%), nitric $(69 \%)$ and phosphoric (86\%) acids and using a calculated value of $\Delta f / \Delta d=5.7 \mathrm{kHz} / \mathrm{um}$ as attained from "Superfish" [7], a frequency decrease of $350 \mathrm{kHz}$ has to occur for $50 \mu \mathrm{m}$ of removal. This corresponds to $=5 \mathrm{~min}$. of chemical polishing at $23^{\circ} \mathrm{C}$. In our surface treatment procedure, pre-chemistry is done in two steps of $2.5 \mathrm{~min}$. each with intermediate rinsing of the cavity to avoid overtheating the acid bath. Subsequently the cavity frequency is measured and if necessary readjusted as well as the field profile. The final chemical treatment of $1 \mathrm{~min}$., corresponding to a frequency change of $-70 \mathrm{kHz}$, is then very predictable and the cold cavity frequency falls within the range of the cold cavity uner of $\pm 200 \mathrm{kHz}$.

\section{B. Pressure Sensitivity between 760 torr and 23 torr}

The cells of CEBAFs 360 cavities are made from highpurity niobium of 3 different suppliers: $3 \%$ were manufactured from W. C. Heraeus niobium, $20 \%$ of the material was supplied by Teledyne and the remainder was manufactured by Fansteel Corp. The room temperature yield strength of this high purity-material was specified to exceed $74 \mathrm{MPa}$. This value varied from manufacturer to manufacturer and from batch to batch. Measurements on amples of the different materials at $300 \mathrm{~K}$ and $4.2 \mathrm{~K}[7]$ also showed significant differences in the yield strength and possible changes in Young's Modulus. Analysis of $\mathbf{4 5}$ cavities showed variations in the pressure sensitivity from $80 \mathrm{~Hz} / \mathrm{Torr}$ to $137 \mathrm{~Hz} / \mathrm{T}$ orr, over each material and from each shipment of material.

\section{CONCLUSIONS}

Analysis of the "as-fabricated" cavity data suggests that the frequency tolerance of $\pm 2 \mathrm{MHz}$ as specified by CEBAF was easily attainable by the manufacturer. The cavity frequency was intentionally kept above the desired 1494.7 $\mathrm{MHz}$ for tuning purposes and the spread was kept within $\pm 1 \mathrm{MHz}$.

Frequency errors in each cell, however, did vary up to several $\mathrm{MHz}$. This variation is probably due to non-uniform spring back in the forming process, machining tolerances and variations in weld shrinkage during electron beam welding. These variations cause different field distributions and require different tuning of each cell resulting in a spread in the-cell-to cell coupling.

The design of the fundamental power coupler (FPC) is such that $Q$ ext of the coupler is sensitive to fabrication tolerances, but the $Q$ of $6.6 \mathrm{E} 6 \pm 20 \%$ can be easily attained with inelastic deformation of the FPC waveguide.

Chemical surface treatment of cavities at CEBAF has rather good reproducibility as long as a stringent control of the bath temperature is maintained. With a cold tuner range of \pm $200 \mathrm{kHz}$ there was no problem obtaining assembled cavities within that range, even though variations in the material properties have resulted in $50 \%$ differences of the sensitivity of the cavities to the helium bath pressure.

Sheet metal technology as applied in the fabrication of the CEBAF cavities without any annealing steps after the mechanical forming operations requires tolerances on the order of $\pm 2 \mathrm{~mm}$ [9]. Tighter tolerances would probably cause an increase in manufacturing costs.

\section{ACKNOWLEDGMENTS}

Many thanks go out to the CEBAF SRF Staff whose efforts have made this paper possible, and a special thanks to K. Yopp who provided CAD suppor.

\section{REFERENCES}

[1] M. Dzenus, et al. "Production of Superconducting Niobium Cavities for CEBAF. "Conference Record of the 1991 Particle Accelerator Conference, p. 2390.

[2] C. Reece, P. Kneisel, J. Mummosser, "Performance of Production Cavities for CEBAF," these proceedings.

[3] P. Kreisel, a al., "Performance of Superconducting Storage Ring Cavities," IEEE Trans. Magn. MAG 21, 1000 (1985).

[4] R. M. Surdelin, private communications.

[5] R. M. Sundelin, "Frequency Spreads Caused by Manufacturing Tolerances," SRF Tech. Note-830102 LNS Comell University.

[6] C. Reece, "Fundemental Power Coupler E-Field Profile Messurements," SRF 831201 LNS Comell University.

[7] K Halbach, R. F. Holsinger, "Superfish: A Compuler Program For Evaluation of R.F. Cavities With Cylindrical Symmetry," Particle Accelerator 1976, Vol. 7, pp 213-222.

[8] P. Kneisel, ef al., "Superconducting Cavities From High Thermal Conducting Niobium For CEBAF," Proceedings of The Conference Electron Bean Melting and Refining, pp. 177 (1990).

[9] J. Benesch, J. Mammosser, "Mochnnical Results of The CEBAF Cenity Series Production," these procoedings. 

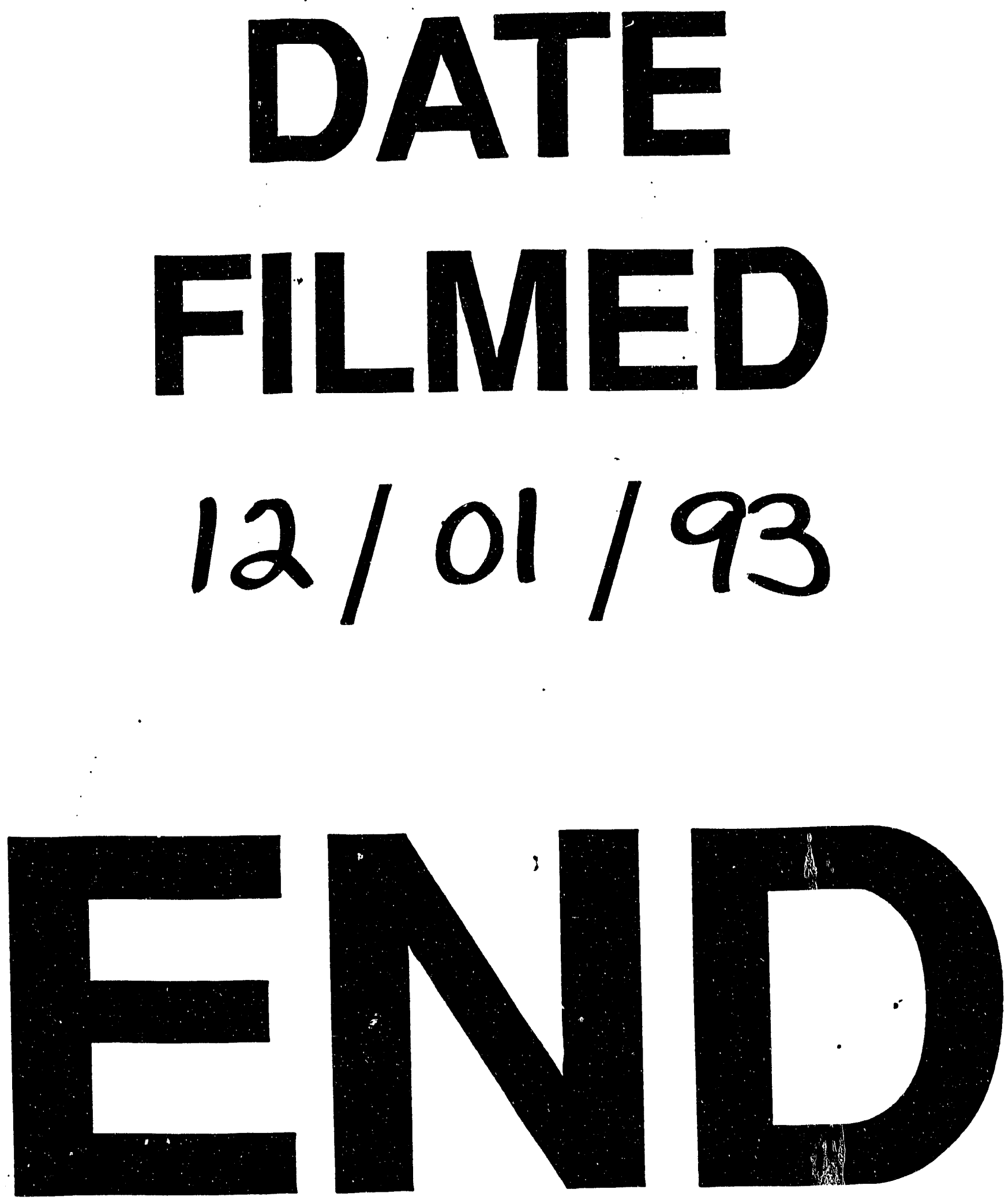
